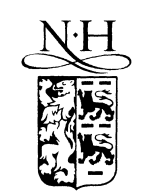

ELSEVIER

\title{
Thermoviscoelasticity of glass-forming liquids
}

\author{
Tage Christensen *, Niels Boye Olsen \\ IMFUFA, Roskilde University Center, Postbox 260, DK-4000 Roskilde, Denmark
}

\begin{abstract}
In supercooled liquids structural relaxation shows up as a frequency dependence of mechanical and thermal properties such as the adiabatic bulk modulus, $K_{\mathrm{s}}$, shear modulus, $G$, expansion coefficient, $\alpha_{\mathrm{p}}$, and isobaric specific heat, $c_{\mathrm{p}}$. Whereas $K_{\mathrm{s}}$ and $G$ can be measured directly, we claim this is difficult for $c_{\mathrm{p}}$. Near the glass transition where $c_{\mathrm{p}}$ differs significantly from the isochoric specific heat, $c_{\mathrm{v}}$, and $G$ approaches $K_{\mathrm{s}}$, heat transport is no longer adequately described by the heat diffusion equation but by four coupled thermoviscoelastic equations. Thus the effective specific heat obtained by specific heat spectroscopy can differ from $c_{\mathrm{p}}$. We present specific heat spectroscopy data on 1,2,4-butanetriol obtained in an approximate spherical symmetric geometry. In this geometry the effect of the thermomechanical coupling can be calculated. The thermal wavelength can be varied from much greater to much smaller than the sample size. Thus the novel method comprises both the homogeneous and inhomogeneous methods. In the homogeneous limit, we, as a good approximation, obtain the pure $c_{\mathrm{p}}$. (c) 1998 Elsevier Science B.V. All rights reserved.
\end{abstract}

\section{Introduction}

It has recently been pointed out [1] that it is questionable whether specific heat spectroscopy measurements on supercooled liquids near the glass transition are truly at isobaric conditions. The temperature perturbation of the calorimetric method gives rise to stresses due to the thermal expansion. The relaxation of these stresses is hindered by the high viscosity near the glass transition. Thus heat transport is no longer adequately described by the heat diffusion equation but by four coupled thermoviscoelastic equations. This effect could explicitly be seen theoretically [1] in the 1-dimensional unilateral case. However the boundary conditions of that example be difficult to achieve in real experiments. It was found that

\footnotetext{
* Corresponding author. Tel.: 4546742 350; fax: 4546743 020; e-mail: tec@ruc.dk.
}

the effective specific heat under such circumstances would be the longitudinal specific heat, $c_{1}=$ $\left(M_{s} / M_{T}\right) c_{\mathrm{v}}$. Here $M_{s}$ and $M_{T}$ are the adiabatic and isothermal longitudinal moduli, respectively, and $c_{\mathrm{v}}$ the isochoric specific heat. We remind that $M$ is related to the bulk modulus, $K$, and shear modulus, $G$, by $M_{s}=K_{s}+\frac{4}{3} G$ (respectively $\left.M_{T}=K_{T}+\frac{4}{3} G\right)$ and that the isobaric specific heat is given by $c_{\mathrm{p}}=\left(K_{s} / K_{T}\right) c_{\mathrm{v}}$. The thermomechanical coupling constant is defined as $\gamma-1=$ $\left(c_{\mathrm{p}}-c_{\mathrm{v}}\right) / c_{\mathrm{v}}=T_{0} \alpha_{P}^{2} K_{s} / c_{\mathrm{p}} \cdot c_{1}$ only equals $c_{\mathrm{p}}$ if the thermomechanical coupling constant is small or if the shear to bulk modulus ratio $G / K_{T}$ is small. However at the glass transition both quantities may be of significant size resulting in a typical deviation $\left(c_{\mathrm{p}}-c_{1}\right) / c_{\mathrm{p}}$ of $20 \%$ based on static values. It could be even worse since we do not know the frequency dependencies of all the other constitutive quantities. Moreover the observed frequency dependence of the specific heat will be distorted by these. In this paper we present some of the 
theoretical results of alternating current calorimetry in the spherical symmetric case and also demonstrate its experimental realization.

\section{Theory}

\subsection{Thermoelasticity}

We describe now the thermal and mechanical interactions between mutual parts of a continuous media and furthermore between the media and the surroundings. To better grasp these somewhat complicated interactions it is fruitful to have a pictorial description besides the mathematical formulation. We will use the so-called energy bond graphs [2-4]. An energy bond is a graphical picture of an energetic interaction between two systems. This interaction is quantified in terms of a generalized force and a generalized current. The energy bond is drawn as a line, the force is depicted by a little bar and the current as an arrow on the en- ergy bond. An energy bond graph of the possible mechanical and thermal action on a volume element is given in Fig. 1. The interactions can be of various tensorial order (scalar, vector, or higher order tensors). The choice of mechanical variables is straight-forward. In the compressional bond the force is the negative of the hydrostatic part of the pressure $-\delta p=\frac{1}{3} \sigma_{k k}$ [5] where $\sigma_{i j}$ is the stress tensor and only deviations from an overall pressure, $p_{0}$, is considered. The corresponding current is the time rate of the relative volume change, $\dot{\delta} \epsilon=\dot{\delta} V / V . \delta \epsilon$ is found from the trace of the strain tensor, $\delta \epsilon=\frac{1}{3} \epsilon_{k k}$ where again the strain tensor is the symmetrized gradient of the displacement field, $\epsilon_{i j}=\frac{1}{2}\left(\partial u_{i} / \partial x_{j}+\partial u_{j} / \partial x_{i}\right)$. The variables of the shear interaction is the deviatoric parts [6] of the stress and strainrate tensors, $s_{i j}=\sigma_{i j}-\frac{1}{3} \sigma_{k k} \delta_{i j}$ and $\dot{e}_{i j}=\dot{\epsilon}_{i j}-\frac{1}{3} \dot{\epsilon}_{k k} \delta_{i j}$. As to the choice of thermal variable Ritmann [7] has argued for the Carnot factor, $1-T_{0} / T$, and the heat current, $\boldsymbol{J}_{q}$, as generalized force and current. Since we are considering small temperature amplitudes the Carnot

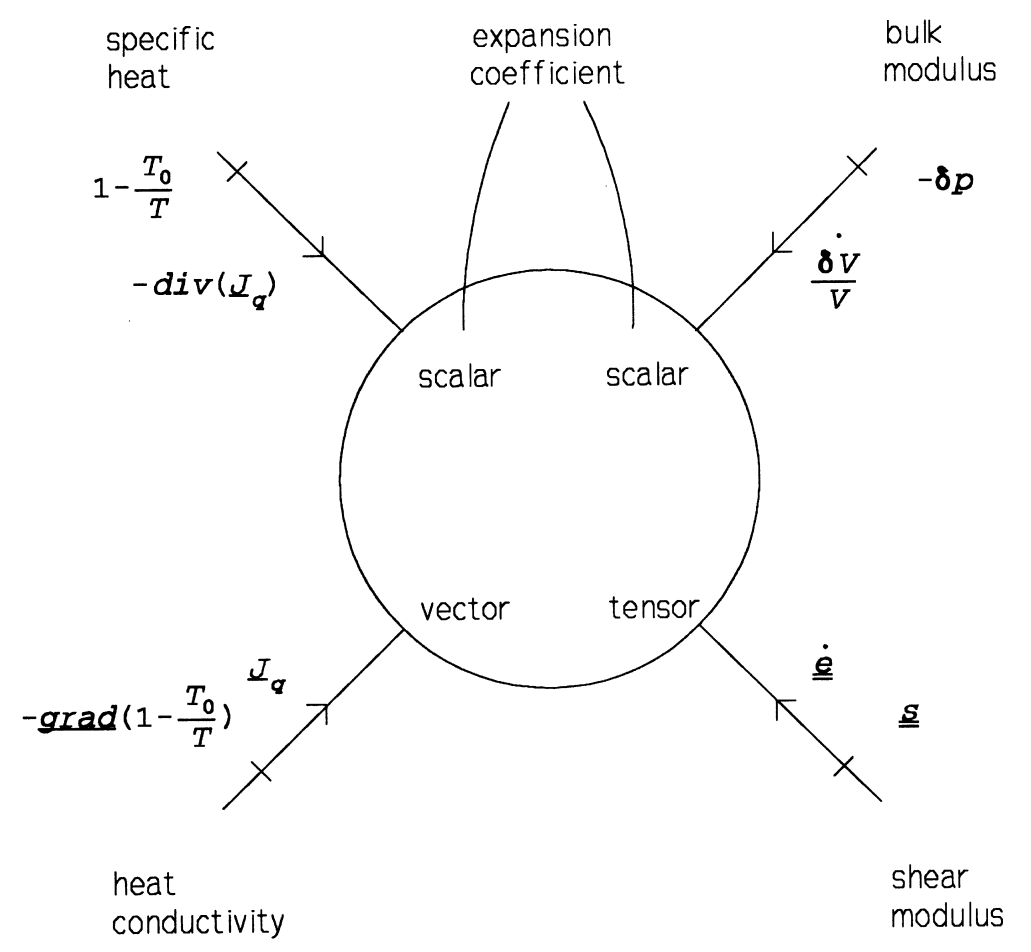

Fig. 1. Energy bond description of the thermal and mechanical interaction between a volume element and its surroundings. 
factor simply becomes the relative temperature change, $\delta T / T_{0}$, where $T_{0}$ is a reference temperature. The Curie-Prigogine principle states that in the linear regime only phenomena of the same tensorial order couples in isotropic systems. Thus the pair, $\delta T / T_{0}$ and $\boldsymbol{J}_{q}$, are not conjugated variables whereas the scalar pair and $\left(\delta T / T_{0}\right.$, $\left.-\operatorname{div}\left(\boldsymbol{J}_{q}\right)\right)$ and vector pair $\left(-\operatorname{grad}\left(\delta T / T_{0}\right), \boldsymbol{J}_{q}\right)$ are, $\dot{q}=-\operatorname{div}\left(\boldsymbol{J}_{q}\right)$ is recognized as the time rate of the supplied heat per volume, $q$. The five thermal and mechanical response functions, the isobaric specific heat, $c_{\mathrm{p}}$, isobaric expansivity, $\alpha_{\mathrm{p}}$, isothermal compressibility, $\kappa_{T}$, heat conductivity, $\lambda$, and shear modulus, $G$, are now expressed in terms of these energy bond variables

$$
\left(\begin{array}{l}
\delta q \\
\delta \epsilon
\end{array}\right)=\left(\begin{array}{ll}
c_{p} & T_{0} \alpha_{p} \\
\alpha_{p} & \kappa_{T}
\end{array}\right)\left(\begin{array}{l}
\delta T \\
-\delta p
\end{array}\right),
$$

$\boldsymbol{J}_{q}=-\lambda \operatorname{grad}(\delta T)$,

$s_{i j}=2 G e_{i j}$.

In a discussion of the relationships between the response functions three levels of description have to be considered. Phenomena on the microscopic molecular level affect all macroscopic response functions giving specific connexions which cannot be deduced from general thermodynamic principles only. From a macroscopic viewpoint (1)-(3) describe a decoupling of some of the properties on the level of a differential volume element. However, on the third level namely in the experimental situation - it is seldom the case that the fields are homogenous and that the sample can be considered as a differential element. This is in particular a problem in the measurements of frequency dependent response functions. The freedom of choosing a suitable heat diffusion length or acoustic wavelength relative to the sample size is lost since they are given by the frequency. Instead we must give a continuum description solving the coupled thermoelastic equations

$$
\begin{gathered}
\left(\frac{4}{3} G+K_{T}\right) \operatorname{grad}(\operatorname{div}(\boldsymbol{u}))-K_{T} \alpha_{p} \operatorname{grad}(T) \\
-G \operatorname{curl}(\operatorname{curl}(\boldsymbol{u}))=\rho \frac{\partial^{2}}{\partial t^{2}} \boldsymbol{u}
\end{gathered}
$$

$c_{\mathrm{v}} \frac{\partial}{\partial t} T+T_{0} K_{T} \alpha_{\mathrm{p}} \frac{\partial}{\partial t} \operatorname{div}(\boldsymbol{u})=\lambda \operatorname{div}(\operatorname{grad}(T))$

with appropriate boundary conditions [8,9]. Then the observable response functions often will not be the pure quantities but instead involve several of these even those separated in the differential element description. One instance of this intricacy is the thermal admittance pr area in a 1-dimensional unilateral geometry [1] which was found to be $Y=\left(-\mathrm{i} \omega c_{l} \lambda\right)^{\frac{1}{2}}$. It thus involves $c_{1}$ and not $c_{\mathrm{p}}$ when a full treatment of the thermoelastic equations is given. The displacement associated with the thermal expansion was, in this case, longitudinal hence the name longitudinal specific heat, $c_{1}$. Another instance is the measurement of the frequency dependent stiffness of a sphere [10] of radius $r$. The stiffness will change from the adiabatic to the isothermal bulk modulus as frequency is varied from much higher to much lower than the characteristic heat diffusion frequency, $\lambda /\left(c_{1} r^{2}\right)$. It will depend on the other response functions in a complicated way in the transition region [11].

\subsection{Thermoviscoelasticity}

It has been implicit in the above discussion that we are dealing with time dependent and not constant response functions which are usually considered in thermoelastic theory [8,9]. This dependency means that Eqs. (4) and (5) and are no longer meaningful in the time domain. However they still hold in the frequency domain where all the response functions become complex. This constitutes the generalization of thermoelasticity into thermoviscoelasticity [12]. All fields are now assumed $\propto e^{\mathrm{i} \omega t}$ and we consider only their complex amplitudes.

\subsection{Spherical symmetry}

Consider a spherical shell of inner radius $r_{1}$ and outer radius $r_{2}$. The energy bond variables at the surface of radius $r$ are the normal component of the stress tensor, $\sigma_{r r}(r)$, abbreviated $\sigma(r)$, the volume swept, $\delta V(r)$, the temperature perturbation, $\delta T(r)$, the heat passed, $Q(r)$. The values of these 
variables at $r_{2}$ can be expressed in terms of the set of values at $r_{1}$ by a transfer matrix

$$
\left(\begin{array}{c}
\sigma \\
\delta T \\
\delta V \\
Q
\end{array}\right)_{r_{2}}=\mathbf{T}\left(r_{2}, r_{1}\right)\left(\begin{array}{c}
\sigma \\
\delta T \\
\delta V \\
Q
\end{array}\right)_{r_{1}} .
$$

One of the advances of the transfer matrix is that a lamination of systems simply correspond to multiplication of the corresponding transfer matrices. Another is that the effect of changing the boundary conditions can readily be found. The explicit expression for $\mathbf{T}$ that can be found from Eqs. (4) and (5) will be given elsewhere [13] but some results derivable from the knowledge of T will be stated here.

\subsubsection{The far inhomogeneous limit}

The inner and outer radii are associated with complex heat diffusion frequencies, $\omega_{1}=\lambda /\left(c_{1} r_{1}^{2}\right)$ and $\omega_{2}=\lambda /\left(c_{1} r_{2}^{2}\right)$. In the far inhomogeneous limit, $\omega \gg\left|\omega_{2}\right|$, the thermal compliance, $J$, seen on the inner surface and defined as $J=Q / \delta T$ becomes

$J=4 \pi\left(\frac{\omega_{1}}{-\mathrm{i} \omega}+\left(\frac{\omega_{1}}{-\mathrm{i} \omega}\right)^{\frac{1}{2}}\right) c_{1} r_{1}^{3}$.

This result is found under both clamped and free mechanical conditions. Under the even stronger condition, $\omega \gg\left|\omega_{1}\right|$, the thermal admittance permit area, $Y=(-\mathrm{i} \omega J) /\left(4 \pi r_{1}^{2}\right)$, then becomes $\left(-\mathrm{i} \omega c_{1} \lambda\right)^{\frac{1}{2}}$ which is expected since the spherical and unilateral case must coincide in this limit.

\subsubsection{The homogeneous limit}

In the homogeneous limit given by $\omega \ll\left|\omega_{2}\right|$ the thermal compliance is dependent on the mechanical boundary conditions. In the case of a free outer boundary, $\sigma\left(r_{2}\right)=0$ and a clamped inner core, $\delta V\left(r_{1}\right)=0$, we find

$J=c_{\mathrm{v}} V\left\{\frac{\left(r_{2} / r_{1}\right)^{3} K_{s}+\frac{4}{3} G}{\left(r_{2} / r_{1}\right)^{3} K_{T}+\frac{4}{3} G}\right\}$,

where $V=\frac{4}{3} \pi\left(r_{2}^{3}-r_{1}^{3}\right)$. Notice that $J \rightarrow c_{1} V$ for $r_{2} / r_{1} \rightarrow 1$ (thin shell) and $J \rightarrow c_{\mathrm{p}} V$ for $r_{2} / r_{1} \rightarrow \infty$ (thick shell). The last fact makes it possible to measure $c_{\mathrm{p}}$.

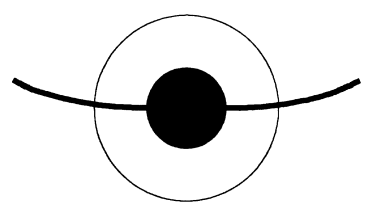

Fig. 2. AC-calorimetry in spherical symmetric geometry. The core of $0.3 \mathrm{~mm}$ diameter is a NTC-resistor with high temperature coefficient. It acts simultaneously as an AC-heat generator and thermometer by the $3 \omega$ technique. The surrounding liquid diameter is $0.7 \mathrm{~mm}$.

\section{Experiment}

The specific heat spectroscopy is accomplished by using a commercial NTC-resistor bead of approximate spherical shape. A droplet of 1,2,4-butanetriol was placed onto the bead as shown in Fig. 2. The thermal admittance is found by a version of the $3 \omega$ technique [14]. The electric resistance of the bead is temperature dependent given by $0.2478 \exp (2656 \mathrm{~K} / T) \Omega$ yielding a temperature coefficient of about $7 \% \mathrm{~K}^{-1}$ near $200 \mathrm{~K}$. The NTC-resistor is connected to a voltage source of cyclic frequency, $\omega / 2$, in series with a temperature independent $100 \mathrm{k} \Omega$ resistor. The Joule heating of the bead gives an $\omega$ component heat current, $\dot{Q}_{\mathrm{NTC}}$, and the in-phase and out-of-phase temperature amplitude is found by the $3 \omega / 2$ component in the voltage of the series resistor. The resolution of the temperature amplitudes are $<1 \mathrm{mK}$ and the stability of the cryostat $\pm 5 \mathrm{mK}$.

\section{Results}

The overall or apparent thermal admittance $Y_{\text {app }}$ is calculated as the ratio of $\dot{Q}_{\mathrm{NTC}}$ to the complex temperature amplitude, $\delta T_{\mathrm{NTC}}$. The apparent heat capacity $C_{\text {app }}$ is defined as $Y_{\text {app }} /(-\mathrm{i} \omega)$ and shown in Fig. 3(a) and (b). The AC-calorimetry which is non-adiabatic is performed under vacuum $\left(<10^{-5}\right.$ torr) to give a suitable small thermal leak between droplet and cryostat. This thermal leak is especially seen in the increase $\propto 1 / \omega$ of the imaginary part of $C_{\text {app }}$ at lower frequencies. The very existence of the peak of $\operatorname{Im} C_{\text {app }}$ is not a consequence of the glass transition. It is present above 

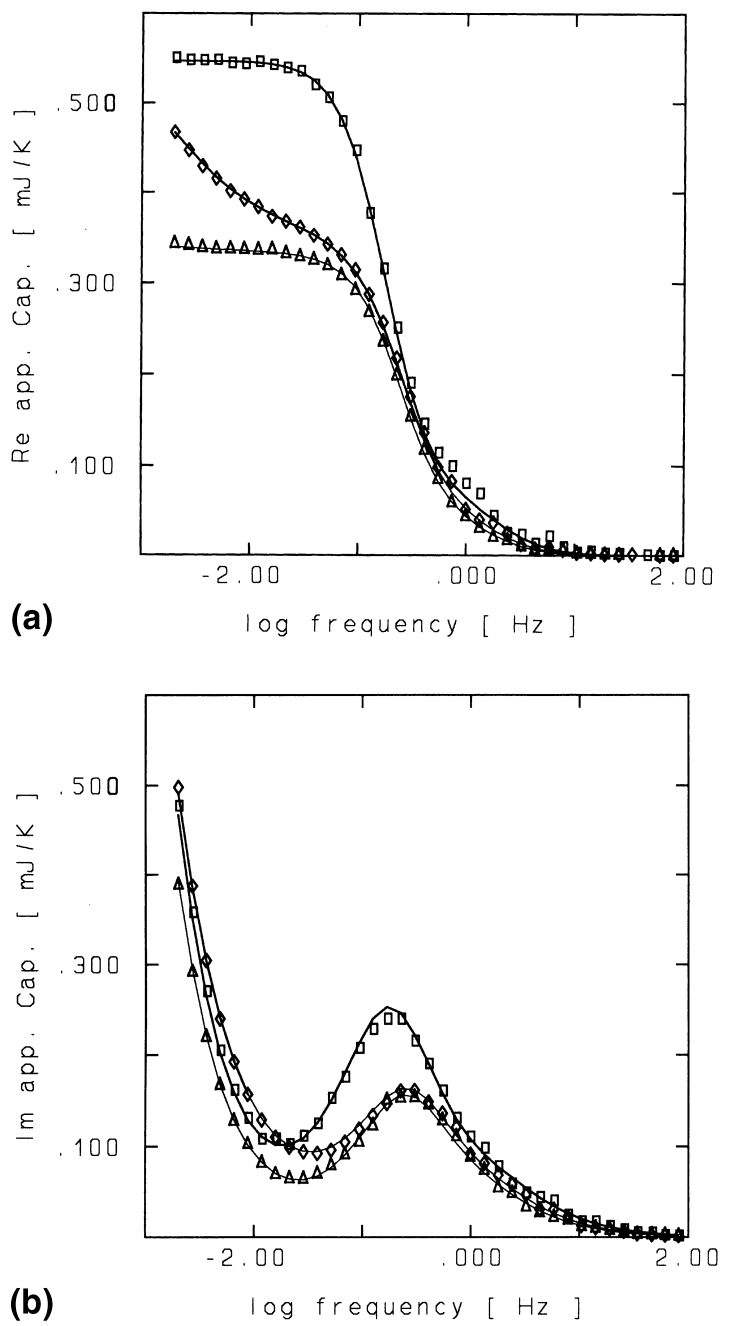

Fig. 3. The apparent heat capacity, $C_{\text {app }}$, as a function of $\log _{10}$ of frequency $\omega /(2 \pi)$. $C_{\text {app }}$ is the ratio of the amplitudes of the heat displacement and temperature at NTC-bead i.e. raw data. Temperatures are $(\square) 211.6 \mathrm{~K},(\diamond) 200.0 \mathrm{~K},(\triangle) 191.4 \mathrm{~K}$. The solid lines are fit to data. (a) At high, $211.6 \mathrm{~K}$ and low, $191.4 \mathrm{~K}$ temperatures where the glass transition is outside the frequency window the real part of $C_{\text {app }}$ is constant in the homogeneous region and decreases on entering the inhomogeneous region. At $200.0 \mathrm{~K}$ the glass transition is seen in the homogeneous regime as a decrease of $\operatorname{Re} C_{\text {app }}$ with frequency. (b) In the imaginary part of $C_{\text {app }}$ the thermal DC-leak contribution is seen at low frequencies. The $200 \mathrm{~K}$-data are above both the $211.6 \mathrm{~K}$ - and 191.4 K-data at low frequencies. This is due to the excess contribution to the dissipative processes from the glass transition. The peak signifies the transition from the homogeneous to the inhomogeneous region and is present irrespective of the glass transition.
(211.6 K) as well as below (191.4 K) the glass transition. It marks the transition from the homogeneous to the inhomogeneous frequency region. The dissipative processes at the glass transition however gives an extra contribution to $\operatorname{Im} C_{\text {app }}$.

\section{Discussion}

To extract the liquid specific heat data from $C_{\text {app }}$ we have to model the thermomechanical interactions between the liquid and its surroundings as depicted in Fig. 4. The NTC-bead is assumed infinitely stiff and to have a negligible expansion coefficient (solid-like). Thus the inner radius of the liquid is clamped whereas the outer is free. Since $\left(r_{2} / r_{1}\right)^{3}=13$ and $G / K_{\mathrm{T}}<1, J$ approximates $c_{\mathrm{p}} V$ well in the homogeneous region (8). A finite stiffness of the bead will only improve this. The reticulation of the thermal part of the composed system in Fig. 4 is given as an electrical equivalent diagram in Fig. 5. The heat resistance of the liquid droplet is called $R_{\mathrm{d}}$. The inner thermal structure of the bead can be modeled by a heat current source, a heat capacitance, $C_{\mathrm{NTC}}$, and heat resistance, $R_{\mathrm{NTC}}$. The value of these and their temperature dependencies are found by measurements on the bare NTC-bead. At $200.0 \mathrm{~K} C_{\mathrm{NTC}}=0.132 \mathrm{~mJ} / \mathrm{K}$ and $R_{\mathrm{NTC}}=150 \mathrm{~K} / \mathrm{W}$. The heat resistance $R$ of the leak is found from the $1 / \omega$-component of $\operatorname{Im~} C_{\text {app }}$ at temperatures above and below the glass transition and interpolated into the transition region. At $200.0 \mathrm{~K} \mathrm{R}=1.87 \times 10^{5} \mathrm{~K} / \mathrm{W}$. According to the nested thermal network of Fig. $5 C_{\text {app }}$ is connected to $J$ by

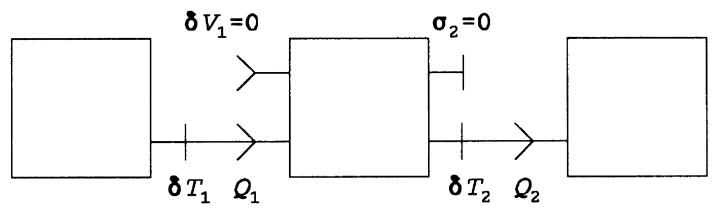

NTC-bead liquid surroundings

Fig. 4. Energy bond graph of the experiment in Fig. 2 in the worst case of clamping by the inner core $\delta V\left(r_{1}\right)=0$. 




Fig. 5. Equivalent diagram corresponding to the thermal interactions of Fig. 4 in the homogenous frequency region. $\dot{Q}_{\mathrm{NTC}}$ generated heat current, $\delta T_{\mathrm{NTC}}$ measured temperature amplitude, $C_{\mathrm{NTC}}$ and $R_{\mathrm{NTC}}$ heat capacity and heat resistance of NTC-bead, $J$ heat capacity of liquid droplet given by Eq. (8), $R_{\mathrm{d}}$ heat resistance of droplet, $R$ heat resistance of leak from droplet to cryostat.

$$
\begin{aligned}
C_{\mathrm{app}}= & \left(-\mathrm{i} \omega R_{\mathrm{NTC}}+\left[C_{\mathrm{NTC}}+\left\{-\mathrm{i} \omega R_{\mathrm{d}}\right.\right.\right. \\
& \left.\left.\left.+\left(J+[-\mathrm{i} \omega R]^{-1}\right)^{-1}\right\}^{-1}\right]^{-1}\right)^{-1} .
\end{aligned}
$$

(Notice this becomes simply $\mathrm{C}_{\mathrm{app}}=(-\mathrm{i} \omega R)^{-1}+$ $C_{\mathrm{NTC}}+J$ when $R_{\mathrm{NTC}}$ and $R_{\mathrm{d}}$ are negligible.) It has earlier been found [10] that the normalized adiabatic bulk modulus of glycerol can be described by $\left(1+s^{-1}+q s^{-\alpha}\right)^{-1}$ which is an extension of the Maxwell model by a scale-invariant term. Here $\alpha, q$ are two shape parameters and $s=-\mathrm{i} \omega \tau(T)$, where $\tau(T)$ is a temperature dependent relaxation time. If the mechanical and thermal relaxations in the liquid are connected as described in Ref. [1] it follows that the normalized isobaric specific heat $\left(\mathrm{c}_{\mathrm{p}}(\omega)-\mathrm{c}_{\mathrm{p}}(\infty)\right) /$ $\left(\mathrm{c}_{\mathrm{p}}(0)-\mathrm{c}_{\mathrm{p}}(\infty)\right)$ is described by a fitting function $\left(1+p^{\prime} s^{1-\alpha}\right) /\left(1+s+p^{\prime} s^{1-\alpha}\right)$. By inserting this expression for $c_{\mathrm{p}}$ times the volume $V$ as $J$ in Eq. (9) and fitting the data of Fig. 3 in the homogeneous region we find at $200.0 \mathrm{~K}: R_{\mathrm{d}}=2.2 \times 10^{2}$ $\mathrm{K} / \mathrm{W}, \quad c_{\mathrm{p}}(0)=2.61 \times 10^{6} \mathrm{~J} / \mathrm{K} / \mathrm{m}^{3}, \quad c_{\mathrm{p}}(\infty)=1.34 \times$ $10^{6} \mathrm{~J} / \mathrm{K} / \mathrm{m}^{3}, \mathrm{p}^{\prime}=0.59, \alpha=0.40$ and $\tau^{\prime}=80 \mathrm{~s}$. The solid lines show the fit. Extension of the fit into the inhomogeneous regime would in principle require the lacking knowledge of, $K_{s}, G(\omega)$ and $\alpha_{\mathrm{p}}(\omega)$. Instead the extension based on putting $G=0$ is shown. This corresponds to assuming $c_{1}$ equals $c_{\mathrm{p}}$ in the far inhomogeneous region. Data is not fitted well at $211.6 \mathrm{~K}$ in the higher frequency tail of the spectrum. It requires higher accuracy than in this preliminary study to judge the significance of this deviation.

\section{Conclusion}

It has been shown that it is possible to do specific heat spectroscopy at the glass transition in a spherical symmetric geometry. The thermal wavelength may, by frequency, be varied from much greater to much smaller than the sample size. Thus the novel method comprises both the homogeneous [15] and inhomogeneous methods [14]. The effect of the thermomechanical coupling can be fully accounted for. The isobaric specific heat can only be obtained directly in a limited frequency range namely the homogeneous range. We have pointed out that AC-calorimetry in different geometries leads to different kinds of specific heat. The seemingly disturbing thermomechanical effect in AC-calorimetry could thus be turned into an additional tool of studying the glass transition.

\section{References}

[1] T. Christensen, N.B. Olsen, Progr. Theoret. Phys. Suppl. 126 (1997) 273.

[2] H.M. Paynter, Analysis and design of engineering systems, MIT, Cambridge, MA, 1960.

[3] D. Karnopp, R.C. Rosenberg, Analysis and Simulation of Multiport Systems - The Bond Graph Approach to Physics Systems Dynamics, MIT, Cambridge, MA, 1968.

[4] P.V. Christiansen, Semiotik og Systemegenskaber (in Danish), IMFUFA text nos. 22 (1979) and 238 (1993), Roskilde.

[5] Einstein summation convention is used.

[6] R.M. Christensen, Theory of Viscoelasticity, Academic Press, New York, 1982.

[7] J. Ritmann, in: J.J. Van Dixhorn, F.J. Evans (Eds.), Physical Structure Systems Theory, Academic Press, London, 1974, p. 35.

[8] L.D. Landau, E.M. Lifshitz, Elasticity Theory, 3rd ed., Course of Theoretical Physics vol.7, Pergamon, New York, 1986.

[9] W. Nowacki, Thermoelasticity, 2nd ed., Pergamon, Oxford, 1986.

[10] T. Christensen, N.B. Olsen, Phys. Rev. Lett. 54 (1985) 2674.

[11] to be published.

[12] J. Jäckle, Physica A 162 (1990) 377.

[13] to be published.

[14] N.O. Birge, S.R. Nagel, Phys. Rev. Lett. 54 (1985) 2674.

[15] T. Christensen, J. Phys. (Paris) Colloq. 46 (1985) C8-635. 